\section{Threshold-based frame error rate analysis of MIMO systems over quasistatic fading channels}

\section{Chatzigeorgiou, I.J. Wassell and R. Carrasco}

Proper selection of a signal-to-noise ratio threshold largely determines the tightness of an approximation to the frame error rate of a system over a quasistatic fading channel. It is demonstrated that the expression for the optimal threshold value, which has been established for singleinput single-output (SISO) channels, remains unchanged for the general case of multiple-input multiple-output (MIMO) channels.

Introduction: In various practical systems, such as fixed wireless access networks, the communication channel experiences extremely slow fading conditions that can be characterised by the quasistatic fading model. In quasistatic fading, the instantaneous signal-to-noise ratio (SNR) at the receiver, denoted as $\gamma$, remains constant for the duration of a frame but changes independently from frame to frame. El Gamal and Hammons [1] demonstrated that the average error probability of an iteratively decoded scheme over a SISO quasistatic fading channel can be accurately approximated by

$$
\tilde{P}_{e}^{Q}\left(\bar{\gamma}, \gamma_{w}\right)=1-e^{-\gamma_{w} / \bar{\gamma}}
$$

where $\bar{\gamma}=E[\gamma]$ is the average SNR and $\gamma_{w}$ is a SNR threshold, based on which the error probability of the iterative decoder is either one or zero depending on whether the instantaneous SNR value is less than or greater than $\gamma_{w}$, respectively. For systems employing turbo codes, $\gamma_{w}$ coincides with the convergence threshold of the iterative decoder $[1,2]$.

Motivated by the work of El Gamal and Hammons [1], Rodrigues et al. [2] and Bouzekri and Miller [3], we demonstrated in [4] that not only iterative but also non-iterative and even uncoded schemes over SISO quasistatic fading channels can also be characterised by a threshold based on which their frame error rate (FER) can be accurately approximated by $\tilde{P}_{e}^{Q}\left(\bar{\gamma}, \gamma_{W}\right)$. In particular, we showed that the SNR threshold is given by

$$
\gamma_{w}=\left(\int_{0}^{\infty} \frac{P_{d}^{G}(\gamma)}{\gamma^{2}} d \gamma\right)^{-1}
$$

where $P_{d}^{G}(\gamma)$ is the probability of successful frame detection in additive white Gaussian noise (AWGN). In this Letter we investigate whether the expression for the SNR threshold still holds in the general case of MIMO quasistatic fading channels.

Preliminaries: We consider a MIMO channel having $N_{T}$ inputs and $N_{R}$ outputs. The transmitter uses space-time block coding [5], while the receiver coherently combines the $N=N_{T} N_{R}$ independent fading paths. If $\gamma$ now corresponds to the instantaneous SNR at the output of the combiner, its probability distribution is given by $[2,6]$

$$
p_{\bar{\gamma}_{R}}(\gamma)=\frac{\gamma^{N-1} e^{-\gamma /\left(\bar{\gamma}_{R} / N_{T}\right)}}{\left(\bar{\gamma}_{R} / N_{T}\right)^{N}(N-1) !}
$$

where $\bar{\gamma}_{R}$ is the average SNR per receive antenna. The approximated FER of the system for MIMO quasistatic fading channels can then be obtained from [2]

$$
\tilde{P}_{e}^{Q}\left(\bar{\gamma}_{R}, \gamma_{w}\right)=\int_{0}^{\gamma_{w}} p_{\bar{\gamma}_{R}}(\gamma) d \gamma=1-e^{-\gamma_{w} N_{T} / \bar{\gamma}_{R}} \sum_{k=0}^{N-1} \frac{\left(\gamma_{w} N_{T} / \bar{\gamma}_{R}\right)^{k}}{k !}
$$

It is important to note that $\tilde{P}_{e}^{Q}\left(\bar{\gamma}_{R}, \gamma_{w}\right)$ is accurate for low to moderate values of $N$; for large $N$, the MIMO channel effectively collapses into an AWGN channel, for which the framework for threshold-based FER analysis does not apply. In the following Section, we derive an exact expression for the optimal SNR threshold $\gamma_{w}$ for the case when (4) can be used to closely approximate the FER of a MIMO system.

SNR threshold evaluation: If an appropriate value for the SNR threshold is selected, we expect the approximated FER of a MIMO system on a quasistatic fading channel to closely represent the exact FER, denoted as $P_{e}^{Q}\left(\bar{\gamma}_{R}\right)$, for a wide range of $\bar{\gamma}_{R}$ values. Ideally,
$\tilde{P}_{e}^{Q}\left(\bar{\gamma}_{R}, \gamma_{w}\right)$ should be identical to $P_{e}^{Q}\left(\bar{\gamma}_{R}\right)$ so that

$$
P_{e}^{Q}\left(\bar{\gamma}_{R}\right)-\tilde{P}_{e}^{Q}\left(\bar{\gamma}_{R}, \gamma_{w}\right)=0
$$

We set $\lambda=N_{T} / \bar{\gamma}_{R}$ and express $P_{e}^{Q}\left(\bar{\gamma}_{R}\right)$ and $\tilde{P}_{e}^{Q}\left(\bar{\gamma}_{R}, \gamma_{w}\right)$ as functions of $\lambda$, that is $P_{e}^{Q}(\lambda)$ and $\tilde{P}_{e}^{Q}\left(\lambda, \gamma_{w}\right)$, respectively. The change of variable will not have an effect on (5). Consequently, the area under the graph of $P_{e}^{Q}(\lambda)$ should be equal to the area under $\tilde{P}_{e}^{Q}\left(\bar{\gamma}, \gamma_{w}\right)$, for $\lambda \in[0 \ldots \Lambda]$ when $\Lambda \rightarrow \infty$. We can thus write

$$
\lim _{\Lambda \rightarrow \infty}\left\{\int_{0}^{\Lambda} P_{e}^{Q}(\lambda) d \lambda-\int_{0}^{\Lambda} \tilde{P}_{e}^{Q}\left(\lambda, \gamma_{w}\right) d \lambda\right\}=0
$$

Taking into account that $P_{e}^{Q}(\lambda)$ can be computed by integrating the FER in AWGN, represented by $P_{e}^{G}(\lambda)$, over the distribution $p_{\lambda}(\gamma)$ given in (3) for $\lambda=N_{T} / \bar{\gamma}_{R}$ [6], we expand the first integral in (6) into

$$
\begin{aligned}
\int_{0}^{\Lambda} P_{e}^{Q}(\lambda) d \lambda & =\int_{0}^{\Lambda} \int_{0}^{\infty} P_{e}^{G}(\gamma) p_{\lambda}(\gamma) d \gamma d \lambda \\
& =\int_{0}^{\Lambda} \int_{0}^{\infty} p_{\lambda}(\gamma) d \gamma d \lambda-\int_{0}^{\Lambda} \int_{0}^{\infty} P_{e}^{G}(\gamma) p_{\lambda}(\gamma) d \gamma d \lambda
\end{aligned}
$$

Note that we have expressed the frame error probability in terms of the probability of successful frame detection in AWGN, that is $P_{e}^{G}(\lambda)=$ $1-P_{d}^{G}(\lambda)$. Substituting $p_{\lambda}(\gamma)$ into $(7)$ gives

$$
\begin{aligned}
\int_{0}^{\Lambda} P_{e}^{Q}(\lambda) d \lambda= & \int_{0}^{\Lambda} \int_{0}^{\infty} \frac{\lambda^{N} \gamma^{N-1}}{(N-1) !} e^{-\lambda \gamma} d \gamma d \lambda \\
& -\int_{0}^{\infty} \frac{P_{d}^{G}(\gamma)}{\gamma(N-1) !} \int_{0}^{\Lambda}(\lambda \gamma)^{N} e^{-\lambda \gamma} d \lambda d \gamma
\end{aligned}
$$

Careful inspection of the function in the double integral reveals that it describes a Poisson distribution; the integral of this probability distribution type from zero to infinity is equal to one. Therefore

$$
\int_{0}^{\Lambda} \int_{0}^{\infty} \frac{\lambda^{N} \gamma^{N-1}}{(N-1) !} e^{-\lambda \gamma} d \gamma d \lambda=\Lambda
$$

Furthermore, if we define

$$
\Omega_{n}(x) \equiv x^{n}+n x^{n-1}+n(n-1) x^{n-2}+\ldots+n !
$$

where $x$ is a real number and $n$ is a positive integer, we can determine that

$$
\int_{0}^{\Lambda}(\lambda \gamma)^{N} e^{-\lambda \gamma} d \lambda=\frac{N !}{\gamma}-\frac{e^{-\Lambda \gamma}}{\gamma} \Omega_{N}(\Lambda \gamma)
$$

based on tables in [7]. Consequently, the first integral in (6) assumes the form

$$
\int_{0}^{\Lambda} P_{e}^{Q}(\lambda) d \lambda=\Lambda-N \int_{0}^{\infty} \frac{P_{d}^{G}(\gamma)}{\gamma^{2}} d \gamma+\int_{0}^{\infty} \frac{P_{d}^{G}(\gamma) \Omega_{N}(\Lambda \gamma)}{\gamma^{2}(N-1) !} e^{-\Lambda \gamma} d \gamma
$$


The second integral in (6) can be evaluated as follows:

$$
\begin{aligned}
-\int_{0}^{\Lambda} \tilde{P}_{e}^{Q}\left(\lambda, \gamma_{w}\right) d \lambda & =-\int_{0}^{\Lambda} 1-e^{-\lambda \gamma_{w}} \sum_{k=0}^{N-1} \frac{\left(\lambda \gamma_{w}\right)^{k}}{k !} d \lambda \\
& =-\Lambda+\sum_{k=0}^{N-1} \frac{1}{k !} \int_{0}^{\Lambda}\left(\lambda \gamma_{w}\right)^{k} e^{-\lambda \gamma_{w}} d \lambda \\
& =-\Lambda+\frac{N}{\gamma_{w}}-\frac{e^{-\lambda \gamma_{w}}}{\gamma_{w}} \sum_{k=0}^{N-1} \frac{\Omega_{k}\left(\Lambda \gamma_{w}\right)}{k !}
\end{aligned}
$$

If we substitute (12) and (13) into (6), we observe that terms $\Lambda$ and $-\Lambda$ cancel each other out. Furthermore, if we take the limit as $\Lambda \rightarrow \infty$, all terms containing $e^{-\Lambda}$ are eliminated since $e^{-\Lambda} \rightarrow 0$. The remaining terms give

$$
-\int_{0}^{\infty} \frac{P_{d}^{G}(\gamma)}{\gamma^{2}} d \gamma+\frac{1}{\gamma_{w}}=0
$$

which can be further reduced to (2); hence, the expression for the SNR threshold of a system is the same for both SISO and MIMO quasistatic fading channels. A practical methodology for the evaluation of $\gamma_{w}$, as well as computational complexity aspects for the proposed thresholdbased analysis, is discussed in [5].

Conclusions: We have considered the general case of a space-time coded system over a MIMO quasistatic fading channel and we have demonstrated that the optimal SNR threshold, for which an approximation to the system's FER is tight, depends on the probability of successful frame detection in AWGN, normalised by the squared SNR.

Acknowledgment: This work was supported by the Engineering and Physical Sciences Research Council (EPSRC) under Grant $\mathrm{EP} / \mathrm{E} 012108 / 1$.
(C) The Institution of Engineering and Technology 2009 6 November 2008

Electronics Letters online no: 20093193

doi: 10.1049/el:20093193

I. Chatzigeorgiou and I.J. Wassell (Computer Laboratory, University of Cambridge, 15 J.J. Thomson Avenue, Cambridge CB3 OFD, United Kingdom)

E-mail: ic231@cantab.net

R. Carrasco (School of Electrical, Electronic and Computer Engineering, Newcastle University, Merz Court, Newcastle upon Tyne NE1 7RU, United Kingdom)

\section{References}

1 El Gamal, H., and Hammons, A.R. Jr.: 'Analyzing the turbo decoder using the Gaussian approximation', IEEE Trans. Inf. Theory, 2001, 47, (2), pp. 671-686

2 Rodrigues, M.R.D., Chatzigeorgiou, I., Wassell, I.J., and Carrasco, R.: 'Performance analysis of turbo codes in quasi-static fading channels', IET Commun., 2008, 2, (3), pp. 449-461

3 Bouzekri, H., and Miller, S.L.: 'An upper bound on turbo codes performance over quasi-static fading channels', IEEE Commun. Lett., 2003, 7, (7), pp. 302-304

4 Chatzigeorgiou, I., Wassell, I.J., and Carrasco, R.: 'On the frame error rate of transmission schemes on quasi-static fading channels'. Proc. CISS, Princeton, NJ, USA, March 2008

5 Slaney, A., and Sun, Y.: 'Space-time coding for wireless communications: an overview', IEE Proc., Commun., 2006, 153, (4), pp. $509-518$

6 Goldsmith, A.: 'Wireless communications' (Cambridge University Press, New York, 2005)

7 Spiegel, M.R., and Liu, J.: 'Schaum's Outlines: mathematical handbook of formulas and tables' (McGraw-Hill, New York, 1999, 2nd edn.) 